\title{
Original
}

\section{The Numerical Analysis of Laplace's Equation in Cylindrical and Hollow Cylindrical Objects with Rotational Symmetry - Applying to Guard-Ring Capacitor -}

\author{
by

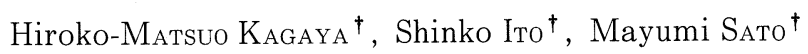 \\ and Toshinobu Soma ${ }^{\dagger}$
}

\begin{abstract}
Fundamental formulations are given for the potential distribution of a cylindrical and a hollow cylindrical objects with a rotational symmetry. This procedure is performed by solving Laplace's equation in polar coordinates using the method of separation of variables. By limiting the inner radius of a hollow cylinder to zero, it can be proved that all the formulations for the hollow cylinder become to those for the cylinder. A few examples of numerical analysis are quantitatively done for the boundary conditions with constant potential value on the cylindrical and hollow cylindrical surface. The obtained data with respect to convergence of a series sum and resultant potential distribution are presented for the different boundary conditions.
\end{abstract}

$\overline{\text { Key Word : Laplace's Equation, Cylinder, Hollow Cylinder, Rotational Sym- }}$ metry, Boundary Condition

\section{Introduction}

The potential problem in cylindrical and hollow cylindrical objects with a rotational symmetry is applied to the charge calculations of several interesting guard-ring capacitors ${ }^{1)}$. The guard-ring capacitor consists of an extensive flat electrode 1 at some distance opposite to a much smaller second electrode 2, which is closely surrounded by an extensive flat electrode 3, called the guard-ring electrode. Two typical configurations of a guard-ring capacitor are shown in Figure 1 (a) and (b). In the volume of the guard-ring capacitor, Laplace's equation is valid. In order to obtain solutions of the potential we devide the axial cross section into areas I and II as shown in Figure 2(a) and (b) corresponding to that in Figure 1 (a) and (b). The subject in Figure 2 are essentially the superposition of the potential problem in cylindrical and hollow cylindrical objects with a rotational symmetry.

Received November 22, 1996

$\dagger$ Department of Computer Science and Engineering, Mining College, Akita University, Akita 010, Japan 


$$
\text { - Applying to Guard-Ring Capacitor - }
$$

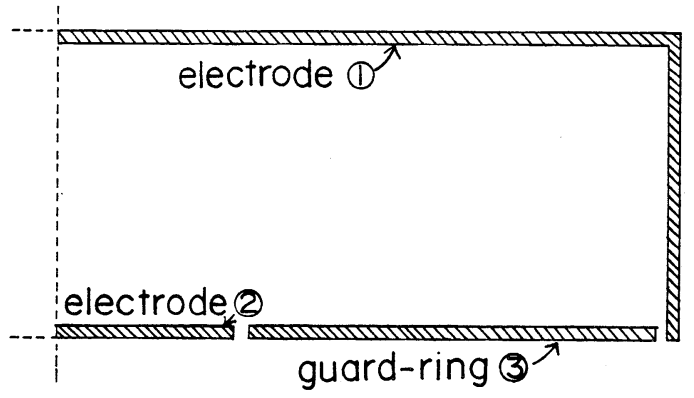

(a)

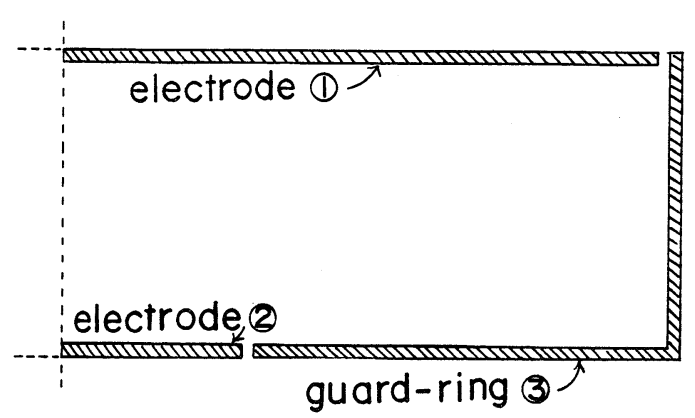

(b)

Figure 1: Axial cross section of two typical configurations of a guard-ring capacitor ; (a) electrode 1 encloses guard-ring 3, and (b) guard-ring 3 encloses electrode 1.

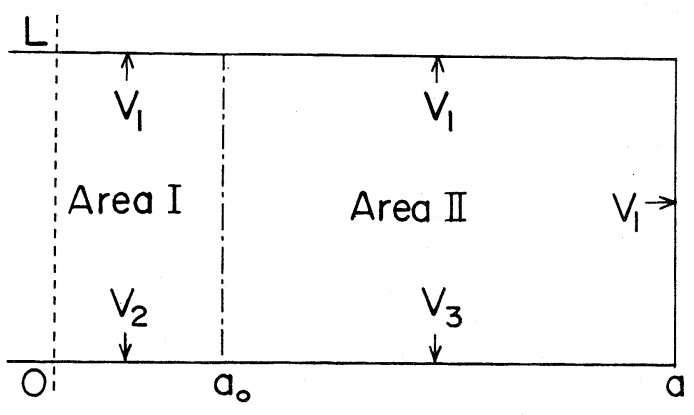

(a)

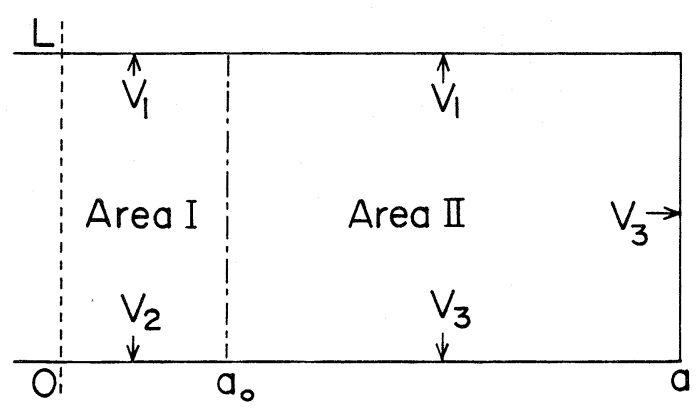

(b)

Figure 2: Axial cross section divided into two areas I and II. Two configurations (a) and (b) correspond to that in Figure 1 (a) and (b).

Laplace's equation in rectangular coordinates has the following form :

$$
\frac{\partial^{2} V}{\partial x^{2}}+\frac{\partial^{2} V}{\partial y^{2}}+\frac{\partial^{2} V}{\partial z^{2}}=0
$$

where $\mathrm{V}$ is the potential and $\mathrm{x}, \mathrm{y}, \mathrm{z}$ are the three rectangular coordinates. By transforming from $(\mathrm{x}, \mathrm{y})$ rectangular coordinates to $(\rho, \phi)$ polar those, eq. (1) become to

$$
\frac{\partial^{2} V}{\partial \rho^{2}}+\frac{1}{\rho} \frac{\partial V}{\partial \rho}+\frac{1}{\rho^{2}} \frac{\partial^{2} V}{\partial \phi^{2}}+\frac{\partial^{2} V}{\partial z^{2}}=0
$$


The boundary conditions have a rotational symmetry so that the potential $\mathrm{V}$ cannot be a function of the coordinate $\phi$. Laplace's equation then reduces to

$$
\frac{\partial^{2} V}{\partial \rho^{2}}+\frac{1}{\rho} \frac{\partial V}{\partial \rho}+\frac{\partial^{2} V}{\partial z^{2}}=0
$$

Using the method of separation of variables, we can express the potential $\mathrm{V}$ as a sum of several products of functions about $\rho$ and $z$ :

$$
V(\rho, z)=\sum R(\rho) Z(z)
$$

where $\mathrm{R}(\rho)$ and $\mathrm{Z}(\mathrm{z})$ can be found by proposing

$$
\frac{d^{2} R}{d \rho^{2}}+\frac{1}{\rho} \frac{d R}{d \rho}+\alpha^{2} R=0 \quad, \quad \frac{d^{2} Z}{d z^{2}}=\alpha^{2} Z
$$

and

$$
\frac{d^{2} R}{d \rho^{2}}+\frac{1}{\rho} \frac{d R}{d \rho}-\beta^{2} R=0 \quad, \quad \frac{d^{2} Z}{d z^{2}}=-\beta^{2} Z
$$

respectively. The solutions of eqs. (5) are given by a combination of normal Bessel functions and hyperbolic functions

$$
V(\rho, z)=\sum_{\alpha}\left(A_{\alpha} \sinh \alpha z+B_{\alpha} \cosh \alpha z\right)\left\{C_{\alpha} J_{0}(\alpha \rho)+D_{\alpha} Y_{0}(\alpha \rho)\right\}
$$

and the solutions of eqs. (6) by a combination of modified Bessel functions ${ }^{2)-4)}$ and trigonometric functions

$$
V(\rho, z)=\sum_{\beta}\left(A_{\beta} \sin \beta z+B_{\beta} \cos \beta z\right)\left\{C_{\beta} I_{0}(\beta \rho)+D_{\beta} K_{0}(\beta \rho)\right\}
$$

The values of the coefficients A, B, C, D and the eigenvalues $\alpha, \beta$ can be found by adapting the final solution $\mathrm{V}(\rho, \mathrm{z})$ to the boundary conditions. Both of eqs. (5) and eqs. (6) with $\alpha=\beta=0$ bring the particular solutions of

$$
\frac{d^{2} R}{d \rho^{2}}+\frac{1}{\rho} \frac{d R}{d \rho}=0 \quad, \quad \frac{d^{2} Z}{d z^{2}}=0
$$

The solutions of eqs. (9) can be given as

$$
V_{0}(\rho, z)=R_{0}(\rho) Z_{0}(z)=\left(A_{0} Z+B_{0}\right)\left(C_{0} \ln \rho+D_{0}\right)
$$



- Applying to Guard-Ring Capacitor-

\section{Laplace's Equation in a Cylindrical Object}

An axial cross section of a cylindrical object with a rotational symmetry is shown in Figure 3.

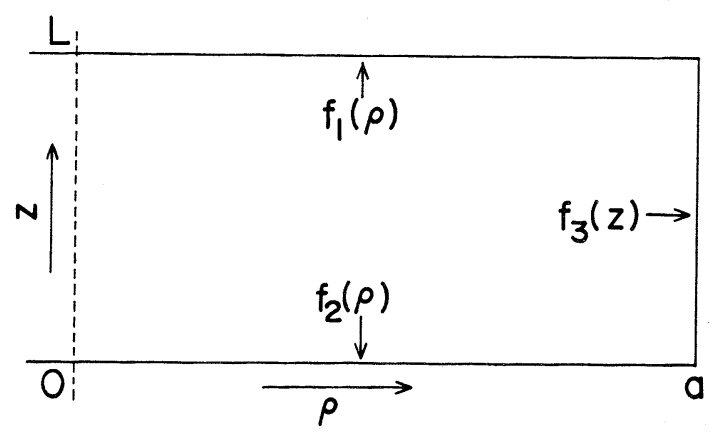

Figure 3: Axial cross section of a cylindrical object with a potential distribution on the cylinder surface.

We assume the following boundary conditions :

$$
z=L ; \quad V=f_{1}(\rho), \quad z=0 ; \quad V=f_{2}(\rho), \quad \rho=a ; \quad V=f_{3}(z)
$$

The required solution $\mathrm{V}(\rho, z)$ in eq. (3) can be given as the superposition of the following three those such as $\mathrm{V}_{1}(\rho, \mathrm{z}), \mathrm{V}_{2}(\rho, \mathrm{z})$ and $\mathrm{V}_{3}(\rho, \mathrm{z})$.

$$
\text { (I) } \quad \frac{\partial^{2} V_{1}}{\partial \rho^{2}}+\frac{1}{\rho} \frac{\partial V_{1}}{\partial \rho}+\frac{\partial^{2} V_{1}}{\partial z^{2}}=0
$$

with

$$
\begin{aligned}
& \rho=a ; V_{1}=0, \quad z=0 ; V_{1}=0, \text { and } z=L ; V_{1}=f_{1}(\rho) . \\
& \text { (II) } \frac{\partial^{2} V_{2}}{\partial \rho^{2}}+\frac{1}{\rho} \frac{\partial V_{2}}{\partial \rho}+\frac{\partial^{2} V_{2}}{\partial z^{2}}=0
\end{aligned}
$$

with

$$
\rho=a ; V_{2}=0, \quad z=0 ; V_{2}=f_{2}(\rho), \text { and } z=L ; V_{2}=0 .
$$




$$
\text { (III) } \quad \frac{\partial^{2} V_{3}}{\partial \rho^{2}}+\frac{1}{\rho} \frac{\partial V_{3}}{\partial \rho}+\frac{\partial^{2} V_{3}}{\partial z^{2}}=0
$$

with

$$
\rho=a ; V_{3}=f_{3}(z), \quad z=0 ; V_{3}=0, \text { and } z=L ; V_{3}=0
$$

In both cases of the boundary condition ( I ) and (II), the coefficient $\mathrm{D}_{\alpha}$ in eq. (7) becomes to zero because Neumann function $\mathrm{Y}_{0}(\alpha \rho)$ diverges at $\rho=0$. Then, the condition $\mathrm{V}_{1 \text { or } 2}=0$ at $\rho=\mathrm{a}$ is satisfied using the zero point $\alpha_{\mathrm{s}}(\mathrm{s}=1,2, \cdots)$ of Bessel function $\mathrm{J}_{0}(\alpha \mathrm{a})$. In the case of the boundary condition (III), the coefficient $\mathrm{D}_{\beta}$ in eq. (8) becomes to zero because the second kind of modified Bessel function $\mathrm{K}_{0}(\beta \rho)$ diverges at $\rho=0$. The final results in the potential equations with eqs. (12) - (17) can be given as follows :

$$
V_{1}(\rho, z)=\sum_{S=1}^{\infty} A_{s} J_{0}\left(\alpha_{s} \rho\right) \sinh \left(\alpha_{s} z\right)
$$

where

$$
\begin{gathered}
A_{S}=\frac{2}{a^{2} \sinh \left(\alpha_{s} L\right)\left\{J_{1}\left(\alpha_{s} a\right)\right\}^{2}} \int_{0}^{a} x f_{1}(x) J_{0}\left(\alpha_{s} x\right) d x, \\
V_{2}(\rho, z)=\sum_{S=1}^{\infty} A_{S} J_{0}\left(\alpha_{S} \rho\right) \sinh \left\{\alpha_{S}(L-z)\right\}
\end{gathered}
$$

where

$$
A_{S}=\frac{2}{a^{2} \sinh \left(\alpha_{S} L\right)\left\{J_{1}\left(\alpha_{s} a\right)\right\}^{2}} \int_{0}^{a} x f_{2}(x) J_{0}\left(\alpha_{s} x\right) d x
$$

and

$$
V_{3}(\rho, z)=\sum_{\mathrm{n}=1}^{\infty} A_{n} I_{0}\left(\frac{n \pi}{L} \rho\right) \sin \left(\frac{n \pi}{L} z\right)
$$

where

$$
A_{n}=\frac{2}{L I_{0}\left(\frac{n \pi}{L} a\right)} \int_{0}^{L} f_{3}(\xi) \sin \left(\frac{n \pi}{L} \xi\right) d \xi
$$

\section{Laplace's Equation in a Hollow Cylindrical Object}

An axial cross section of a hollow cylindrical object with a rotational symmetry is shown in 


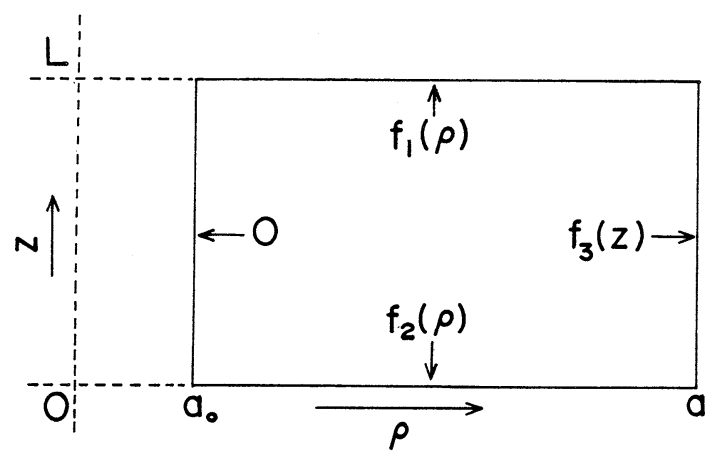

Figure 4: Axial cross section of a hollow cylindrical object with a potential distribution on the hollow cylinder surface.

Figure 4. We assume the following boundary conditions :

$$
\begin{aligned}
& \quad \begin{array}{l}
\quad z=L ; V=f_{1}(\rho), \quad z=0 ; V=f_{2}(\rho), \quad \rho=a_{0} ; V=0 \\
\text { and } \\
\quad \rho=a ; V=f_{3}(z) .
\end{array}
\end{aligned}
$$

The required solution $\mathrm{V}(\rho, z)$ in eq. (3) can be given as the superposition of the following three those with the corresponding boundary conditions such as $\mathrm{V}_{1}(\rho, z), \mathrm{V}_{2}(\rho, z)$ and $\mathrm{V}_{3}(\rho, \mathrm{z})$.

$$
\text { (I) } \quad \frac{\partial^{2} V_{1}}{\partial \rho^{2}}+\frac{1}{\rho} \frac{\partial V_{1}}{\partial \rho}+\frac{\partial^{2} V_{1}}{\partial z^{2}}=0
$$

with

$$
\begin{gathered}
\rho=a_{0} ; V_{1}=0, \quad \rho=a ; V_{1}=0, \quad z=0 ; V_{1}=0 \text { and } z=L ; V_{1}=f_{1}(\rho) \\
\text { (II ) } \frac{\partial^{2} V_{2}}{\partial \rho^{2}}+\frac{1}{\rho} \frac{\partial V_{2}}{\partial \rho}+\frac{\partial^{2} V_{2}}{\partial z^{2}}=0
\end{gathered}
$$

with

$$
\begin{gathered}
\rho=a_{0} ; V_{2}=0, \quad \rho=a ; V_{2}=0, \quad z=0 ; V_{2}=f_{2}(\rho) \\
\text { and } z=L ; V_{2}=0 . \\
\text { (III) } \frac{\partial^{2} V_{3}}{\partial \rho^{2}}+\frac{1}{\rho} \frac{\partial V_{3}}{\partial \rho}+\frac{\partial^{2} V_{3}}{\partial z^{2}}=0
\end{gathered}
$$


with

$$
\begin{gathered}
\rho=a_{0} ; V_{3}=0, \quad \rho=a ; V_{3}=f_{3}(z), \quad z=0 ; \quad V_{3}=0 \\
\text { and } z=L ; V_{3}=0 .
\end{gathered}
$$

In both cases of the boundary condition ( I ) and (II), the condition $\mathrm{V}_{1_{\text {or } 2}}=0$, at $\rho=0$ and $\rho=$ a is satisfied using the zero point $\alpha_{\mathrm{s}}(\mathrm{s}=1,2, \cdots)$ of the definite function $\mathrm{W}\left(\alpha \mathrm{a}, \alpha \mathrm{a}_{0}\right)$ where $\mathrm{W}(\mathrm{x}, \mathrm{y})=\mathrm{J}_{0}(\mathrm{x}) \mathrm{Y}_{0}(\mathrm{y})-\mathrm{J}_{0}(\mathrm{y}) \mathrm{Y}_{0}(\mathrm{x})$. Then, in the case of the boundary condition (III), the condition $\mathrm{V}_{3}=0$ at $\rho=\mathrm{a}_{0}$ is satisfying using the specific function $\mathrm{W}^{\prime}(\mathrm{x}, \mathrm{y})=\mathrm{I}_{0}(\mathrm{x}) \mathrm{K}_{0}(\mathrm{y})-\mathrm{I}_{0}(\mathrm{y})$ $\mathrm{K}_{0}(\mathrm{x})$. The final solutions in the potential equations with eqs. (25) - (30) can be given as follows.

$$
V_{1}(\rho, z)=\sum_{\mathrm{S}=1}^{\infty} A_{S} W\left(\alpha_{s} \rho, \alpha_{s} a_{0}\right) \sinh \left(\alpha_{s} z\right)
$$

where

$$
\begin{gathered}
A_{S}=\frac{1}{\sinh \left(\alpha_{s} L\right)} \int_{a_{0}}^{a} x W\left(\alpha_{S} x, \alpha_{s} a_{0}\right) f_{1}(x) d x / \int_{a_{0}}^{a} x\left\{W\left(\alpha_{S} x, \alpha_{s} a_{0}\right)\right\}^{2} d x, \\
V_{2}(\rho, z)=\sum_{S=1}^{\infty} A_{S} W\left(\alpha_{S} \rho, \alpha_{s} a_{0}\right) \sinh \left\{\alpha_{S}(L-z)\right\},
\end{gathered}
$$

where

$$
A_{S}=\frac{1}{\sinh \left(\alpha_{s} L\right)} \int_{a_{0}}^{a} x W\left(\alpha_{s} x, \alpha_{s} a_{0}\right) f_{2}(x) d x / \int_{a_{0}}^{a} x\left\{W\left(\alpha_{s} x, \alpha_{s} a_{0}\right)\right\}^{2} d x,
$$

and

$$
V_{3}(\rho, z)=\sum_{n=1}^{\infty} A_{n} \sin \left(\frac{n \pi}{L} z\right) W^{\prime}\left(\frac{n \pi}{L} \rho, \frac{n \pi}{L} a_{0}\right) / W^{\prime}\left(\frac{n \pi}{L} a, \frac{n \pi}{L} a_{0}\right)
$$

where

$$
A_{n}=\frac{2}{L} \int_{0}^{L} \sin \left(\frac{n \pi}{L} \xi\right) f_{3}(\xi) d \xi
$$

Finally, we consider a few relations for Bessel and modified Bessel functions ${ }^{2)-4}$. If the argument $\mathrm{x}$ becomes zero the following expressions are valid :

$$
x \rightarrow 0: J_{0}(x) \rightarrow 1, \quad Y_{0}(x) \rightarrow-\infty, \quad I_{0}(x) \rightarrow 1, \quad K_{0}(x) \rightarrow \infty
$$

and 


$$
\frac{W\left(x_{1}, x\right)}{W\left(x_{2}, x\right)} \rightarrow \frac{J_{0}\left(x_{1}\right)}{J_{0}\left(x_{2}\right)}, \frac{W^{\prime}\left(x_{1}, x\right)}{W^{\prime}\left(x_{2}, x\right)} \rightarrow \frac{I_{0}\left(x_{1}\right)}{I_{0}\left(x_{2}\right)}
$$

If we let the inner radius $\mathrm{a}_{0}$ decreases to zero, it can be proved easily with the aid of eqs. (37) that all the solutions for a hollow cylindrical object such as eqs. (31) - (36) change into those for a cylindrical one such as eqs. (18) - (23).

\section{A Few Examples of Numerical Analysis}

As the basic application of numerical solution, we adopt the boundary conditions with constant potential value on the cylindrical and hollow cylindrical surface. First, we apply to Laplace's equation in cylindrical object. Substituting $\mathrm{f}_{1}(\rho)=\mathrm{c}_{1}$ into eq. (19) for the boundary condition (I), we can practise the integration over Bessel function and obtain

$$
A_{s}=\frac{2 c_{1}}{a \alpha_{s} \sinh \left(\alpha_{s} L\right) J_{1}\left(\alpha_{s} a\right)}
$$

In Figure 5, we show the convergence of a series sum with eq. (18) at $\rho / \mathrm{a}=0.5$ and $\mathrm{z} / \mathrm{L}=0.9,0.8$. We select $\mathrm{L} / \mathrm{a}=1$ and 0.1 as the representative size of the cylinder. From Figure 5, we see that the convergence of a series sum becomes slow as the size of $L$ becomes small. In Figure 6, we show the

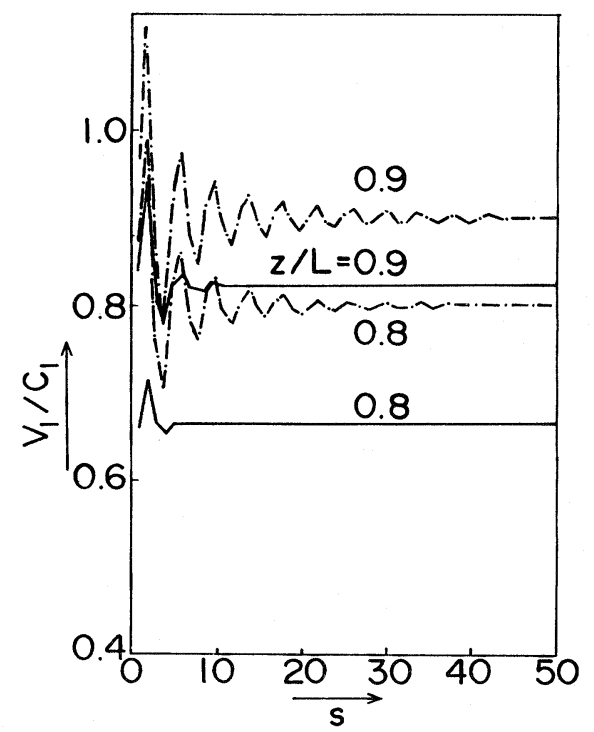

Figure 5: Convergence of a series sum with eq. (18) at $\rho / \mathrm{a}=0.5$ and $\mathrm{z} / \mathrm{L}=0.9,0.8$. Full curves correspond to those for $\mathrm{L}$ $/ \mathrm{a}=1$ and dotted curves for $\mathrm{L} / \mathrm{a}=$ 0.1 in what follows.

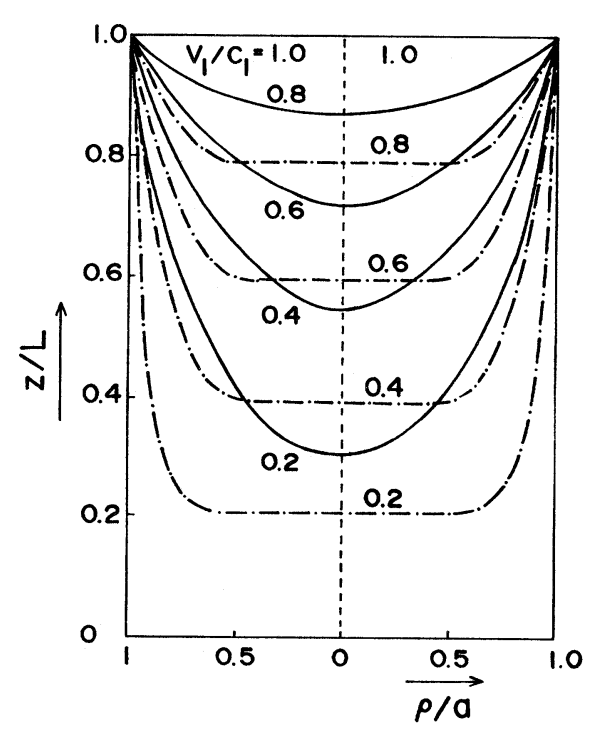

Figure 6: Obtained potential distribution $\mathrm{V}_{1}$ of a cylindrical object with a rotational symmetry for the boundary condition ( I ) in eqs.(13) and (19) with $\mathrm{f}_{1}(\rho)=c_{1}$. 
obtained potential distribution $V_{1} / c_{1}$ of a cylindrical object for the boundary condition ( I ). Because of a rotational symmetry, the constant potential curves in Figure 6 are symmetric with respect to the central axis with $\rho=0$, and the resultant data are given in semi cross section involving central axis in what follows. In Figure 6 , we see that the potential distribution $V_{1} / c_{1}$ is more diffused as the size of $\mathrm{L}$ becomes small. Because the potential distribution $\mathrm{V}_{2} / \mathrm{c}_{2}$ for the boundary condition ( II ) is symmetric to that $\mathrm{V}_{1} / \mathrm{c}_{1}$ for the boundary condition ( I ) with respect to the line with $\mathrm{z}=\mathrm{L} / 2$, we omit the resultant data for the boundary condition (II) in what follows.

Substituting $f_{3}(z)=c_{3}$ into eq. (23) for the boundary condition (III), we can practise the integration over trigonometric function and obtain

$$
A_{n}=\frac{2 c_{3}}{n \pi I_{0}\left(\frac{n \pi}{L} a\right)}\left\{1-(-1)^{n}\right\}
$$

In Figure 7, we show the convergence of a series sum with eq. (22) at $\mathrm{z} / \mathrm{L}=0.5$. In Figures 8 (a) and (b), we show the obtained potential distribution $\mathrm{V}_{3} / \mathrm{c}_{3}$ of a cylindrical object for the boundary condition (III). In Figure 8 (a), we note that the potential value $V_{3} / c_{3}$ is non-zero along central axis with $\rho=0$. From Figures 7 and 8 , we see that the convergence of a series sum with eq. (22) becomes rapid and that the potential distribution $\mathrm{V}_{3} / \mathrm{c}_{3}$ is more concentrated on the side wall with $\rho=\mathrm{a}$ as the size of L becomes small.

Second, we apply to Laplace's equation in hollow cylindrical object. As typical examples of several interesting guard-ring capacitors ${ }^{1)}$ with $\left(a-a_{0}\right) \gtrsim 5 L$, we select $a_{0} / a=0.25$ and $L /\left(a-a_{0}\right)$ $=0.2,0.1$ for the representative size of the hollow cylinder. In addition, we adopted $\mathrm{a}_{0} / \mathrm{a}=0.5$ and $\mathrm{L} /\left(\mathrm{a}-\mathrm{a}_{0}\right)=0.2,0.1$ and the resultant potential distribution was almost as same as the former with $a_{0} / a=0.25$. Substituting $f_{1}(\rho)=c_{1}$ into eq. (32) for the boundary condition (I), we obtain

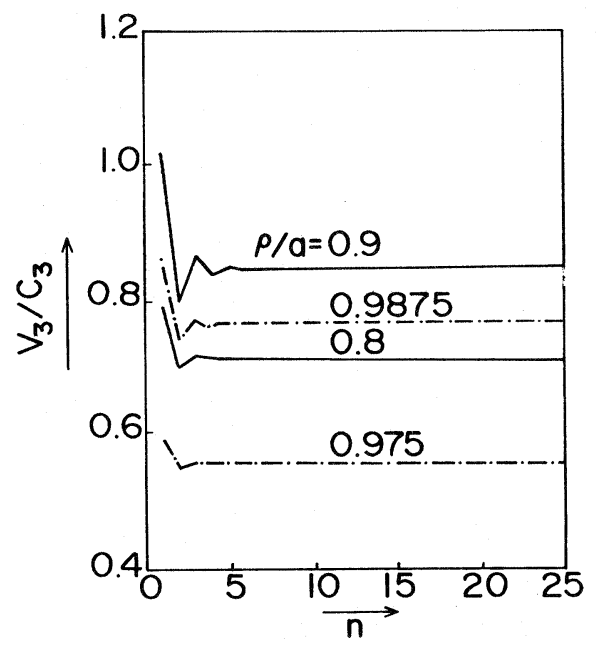

Figure 7: Convergence of a series sum with eq. (22) at $\mathrm{z} / \mathrm{L}=0.5$. 

- Applying to Guard-Ring Capacitor -

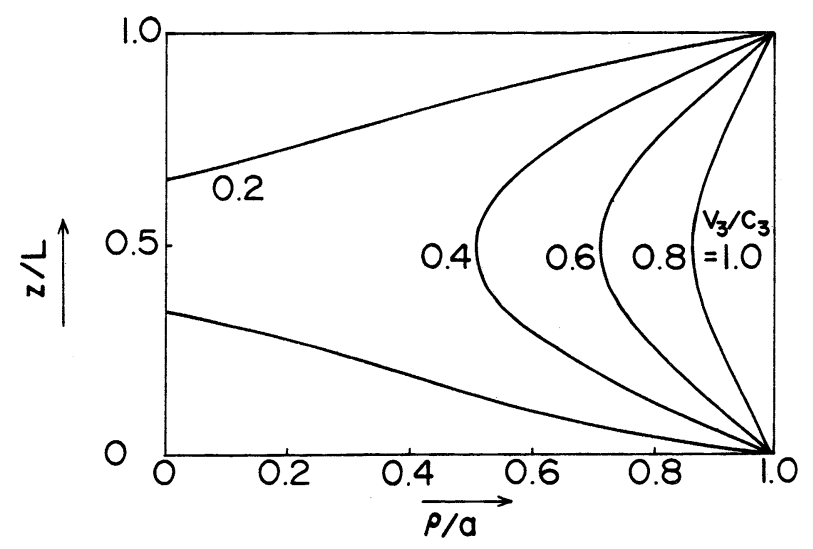

(a)

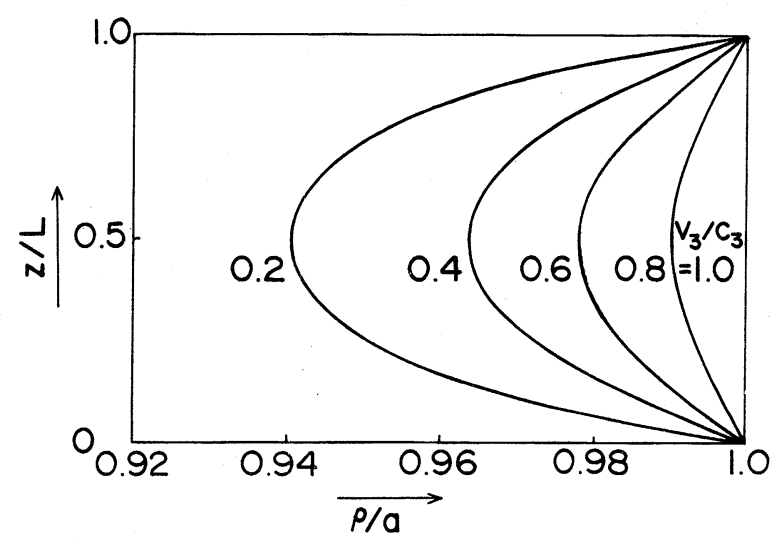

(b)

Figure 8: Obtained potential distribution $\mathrm{V}_{3}$ of a cylindrical object for the boundary condition (III) in eqs. (17) and (23) with $\mathrm{f}_{3}(\mathrm{z})=\mathrm{c}_{3}$. (a) $\mathrm{L} / \mathrm{a}=1$ and (b) $\mathrm{L} / \mathrm{a}=$ 0.1 .

$$
A_{S}=\frac{c_{1}}{\sinh \left(\alpha_{S} L\right)} \int_{a_{0}}^{a} x W\left(\alpha_{S} x, \alpha_{S} a_{0}\right) d x / \int_{a_{0}}^{a} x\left\{W\left(\alpha_{S} x, \alpha_{S} a_{0}\right)\right\}^{2} d x
$$

where the numerical analysis over the integration is practised. In Figure 9, we show the convergence of a series sum with eq. (31) at $\rho / a=\left(a+a_{0}\right) /(2 a)$ and $z / L=0.8,0.5$. From Figure 9, we see that the convergence of a series sum becomes somewhat slow as the size of $\mathrm{L}$ becomes small. In 


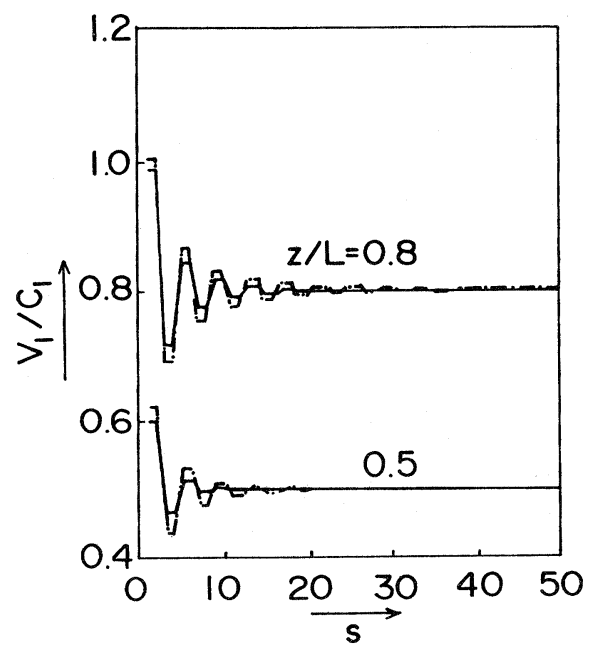

Figure 9: Convergence of a series sum with eq. (31) at $\rho / \mathrm{a}=\left(\mathrm{a}+\mathrm{a}_{0}\right) /(2 \mathrm{a})$ for $\mathrm{a} / \mathrm{a}_{0}$ $=0.25$. Full curves correspond to those for $\mathrm{L} /\left(\mathrm{a}-\mathrm{a}_{0}\right)=0.2$ and dotted curves for $L /\left(a-a_{0}\right)=0.1$ in what follows.

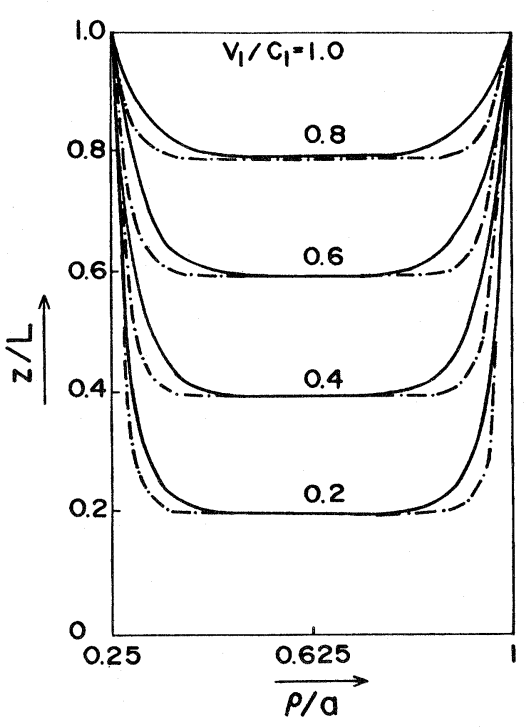

Figure 10: Obtained potential distribution $\mathrm{V}_{1}$ of a hollow cylindrical object for the boundary condition ( I ) in eqs. (26) and (32) with $\mathrm{f}_{1}(\rho)=\mathrm{c}_{1}$.

Figure 10, we show the obtained potential distribution $V_{1} / c_{1}$ of a hollow cylindrical object for the boundary condition (I). From Figure 10, we see that the potential distribution $V_{1} / c_{1}$ is more diffused near the both side wall with $\rho=\mathrm{a}_{0}$ and a as the size of $\mathrm{L}$ becomes small.

Substituting $\mathrm{f}_{3}(\mathrm{z})=\mathrm{c}_{3}$ into eq. (36) for the boundary condition (III), we can practise the integration over trigonometric function and obtain

$$
A_{n}=\frac{2 c_{3}}{n \pi}\left\{1-(-1)^{n}\right\}
$$

In Figure 11, we show the convergence of a series sum with eq. (35) at $\mathrm{z} / \mathrm{L}=0.5$ and $\rho / \mathrm{a}=0.99,0.98$. In Figures 12 (a) and (b), we show the obtained potential distribution $\mathrm{V}_{3} / \mathrm{c}_{3}$ of a hollow cylindrical object for the boundary condition (III). From Figures 11and 12, we see that the convergence of a series sum with eq. (35) becomes somewhat rapid and that the potential distribution $\mathrm{V}_{3} / \mathrm{c}_{3}$ is more concentrated on the side wall with $\rho=$ a as the size of $L$ becomes small.

Last, by limiting the inner radius a to zero, we discuss numerically the Laplace's solution from hollow cylinder to cylinder. In Figures 13 and 14 , we show the resultant potential distribution $\mathrm{V}_{1} / \mathrm{c}_{1}$ and $\mathrm{V}_{3} / \mathrm{c}_{3}$ of a cylindrical object from the limiting case $\mathrm{a}_{0} \rightarrow 0$ of a hollow cylindrical one for the boundary condition condition (I ) with $\mathrm{f}(\rho)=\mathrm{c}_{1}$ and (III) with $\mathrm{f}_{1}(\mathrm{z})=\mathrm{c}_{3}$ for $\mathrm{L} / \mathrm{a}=1$. From Figures 13 and 14, we see that that the potential distribution near central axis with $\rho=0$ is largely changed in comparison with the corresponding those such as in Figures 6 and 8 (a). This 


$$
\text { - Applying to Guard-Ring Capacitor - }
$$

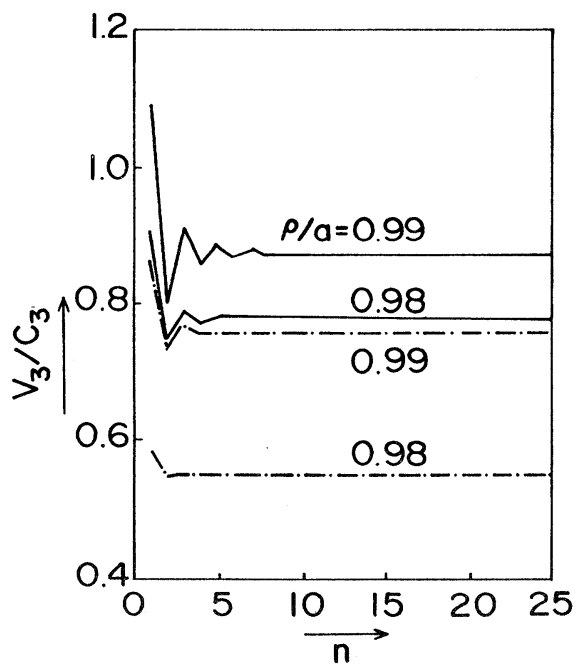

Figure 11: Convergence of a series sum with eq. (35) at $\mathrm{z} / \mathrm{L}=0.5$ and $\rho / \mathrm{a}=0.99,0.98$ for $\mathrm{a} / \mathrm{a}_{0}=0.25$.

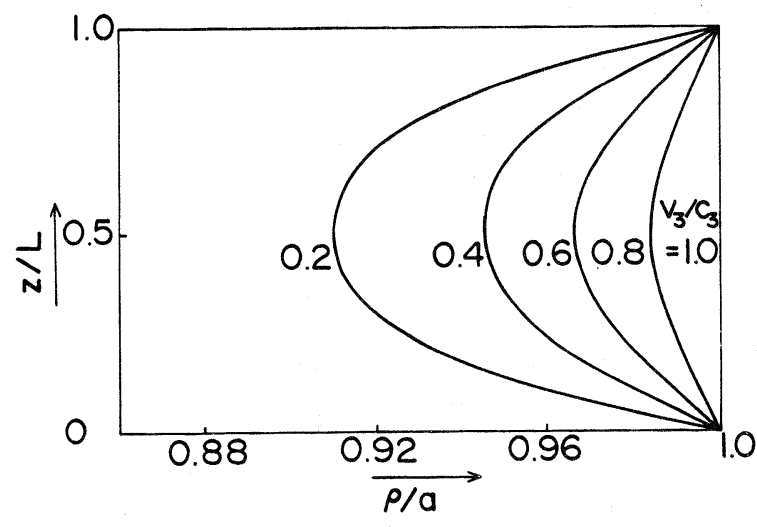

(a)

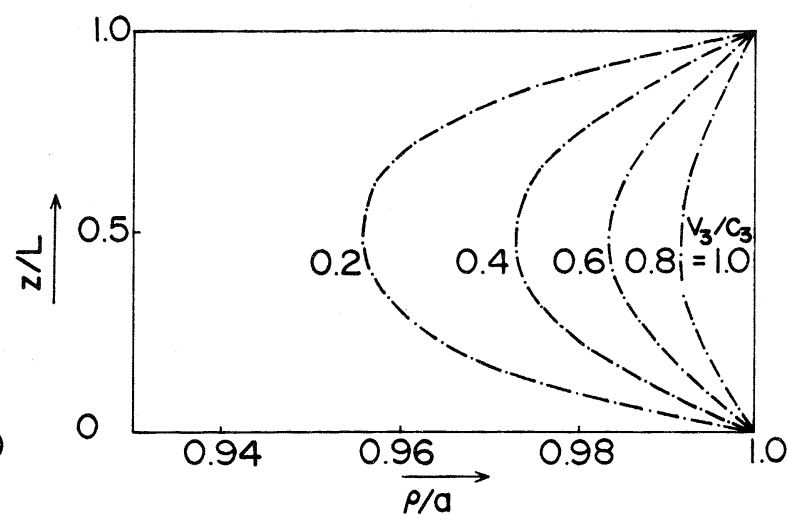

(b)

Figure 12: Obtained potential distribution $\mathrm{V}_{3}$ of a hollow cylindrical object for the boundary condition (III) in eqs. (30) and (36) with $\mathrm{f}_{3}(\mathrm{z})=\mathrm{c}_{3}$. (a) $\mathrm{L} /\left(\mathrm{a}-\mathrm{a}_{0}\right)=0.2$ and (b) $\mathrm{L} /\left(\mathrm{a}-\mathrm{a}_{0}\right)=0.1$. 


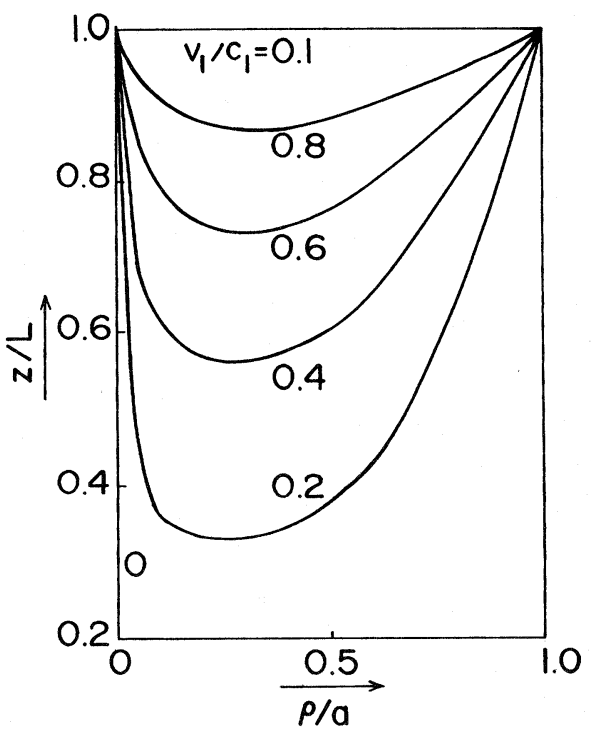

Figure 13: Resultant potential distribution $\mathrm{V}_{1}$ of a cylindrical object from the limiting case $\mathrm{a}_{0} \rightarrow 0$ of a hollow cylindrical one for the boundary condition (I) with $\mathrm{f}_{1}(\rho)=\mathrm{c}_{1}$ for $\mathrm{L} / \mathrm{a}=1$.

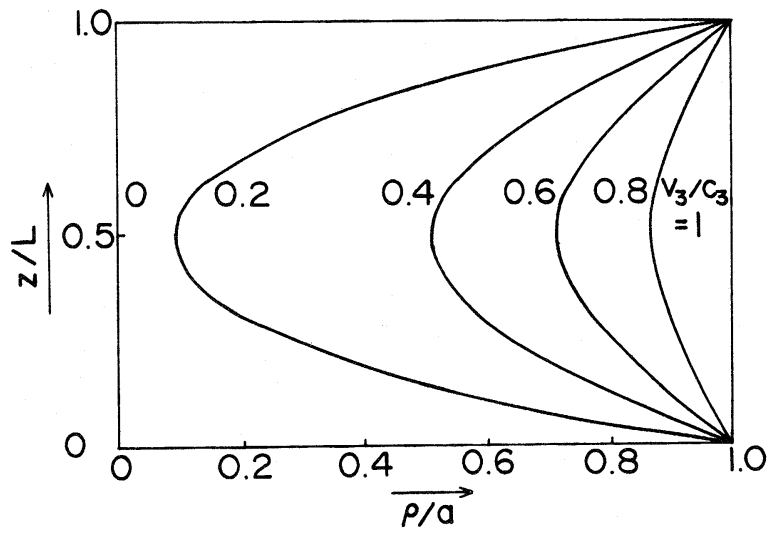

Figure 14: Resultant potential distribution $\mathrm{V}_{3}$ of a cylindrical object from the limiting case $a_{0} \rightarrow 0$ of a hollow cylindrical one for the boundary condition (III) with $\mathrm{f}_{3}(\mathrm{z})=\mathrm{c}_{3}$ for $\mathrm{L} / \mathrm{a}=1$.

contradictory results are due to the introduction of an extra boundary condition such as $\mathrm{V}=0$ at $\rho=$ $\mathrm{a}_{0}$ for a hollow cylidrical object in section 3 , and may be overcome by imposing the non-zero potential values in Figures 6 and 8 (a). 


\section{Conclusion}

In the present work, we presented fundamental formulations for the potential distribution of a cylindrical and a hollow cylindrical objects with a rotational symmetry. Then, we applied to the numerical solution for the boundary conditions with constant potential value on the cylindrical and hollow cylindrical surface. We obtained the useful data with respect to the convergence of a series sum, resultant distribution for the different boundary conditions and the transformation of the Laplace's equation from hollow cylinder to cylinder. The obtained data are important to apply directly to the charge distribution of several interesting guard-ring capacitors and to study the electric properties of the material.

\section{Acknowledgement}

The authors would like to thank Mr. K. Takagi for help in performing the preliminary calculations. The numerical calculations were carried out with the ACOS 3900 operating system in the Computer Center of Tohoku University.

\section{References}

1) W. Chr. Heerens and F. C. Vermeulen, J. Appl. Phys. 46, 2486 (1975).

2) I. N. Sneddon, Special Functions of Mathematical Physics and Chemistry (Oliver and Boyd, London, 1966).

3) F. E. Relton, Applied Bessel Functions (Dover, New York, 1965).

4) G. N. Watson, Theory of Bessel Functions (Cambridge U. P., Cambridge, 1952). 